Steve M. Hollenberg

\title{
Think locally: evaluation of the microcirculation in sepsis
}

Received: 28 May 2010

Accepted: 30 May 2010

Published online: 20 August 2010

(C) Copyright jointly held by Springer and ESICM 2010

\section{S. M. Hollenberg (凶)}

Division of Cardiovascular Diseases, Cooper University Hospital, 1 Cooper Plaza, 366 Dorrance, Camden, NJ 08034, USA

e-mail: Hollenberg-Steven@ CooperHealth.edu

Tel.: +1-856-9687636

Fax: +1-856-9687420

The ultimate goals of hemodynamic therapy in sepsis are to restore effective tissue perfusion and to maintain cellular metabolism. Fluid resuscitation is the first step in management of septic shock. Fluid administration should be performed vigorously and titrated to clinical endpoints of perfusion such as capillary refill, urine output, and mental status, and also to macrocirculatory parameters of global perfusion, including heart rate, blood pressure, cardiac output, and mixed or central venous oxygen saturation [1]. In sepsis, however, tissue hypoperfusion may result not only from decreased perfusion pressure attributable to hypotension but also from abnormal distribution of blood flow [1,2]. Thus, defining the adequacy of resuscitation requires attention to both global and regional perfusion. The microcirculation is a critical component of the cardiovascular system that regulates flow to the tissues. Microcirculatory perturbation is a central abnormality in septic shock and represents a logical and promising therapeutic target [3].

In this context, an interesting manuscript in the current issue of Intensive Care Medicine, along with one published in the June 2010 issue, provide useful new information. In this issue, Pottecher et al. [4] compare the microcirculatory effects of passive leg raising and volume expansion in septic patients using a sidestream dark field
(SDF) imaging device to assess the sublingual circulation. The patients were predicted to be preload-responsive, as assessed by pulse pressure variation greater than 13\% [5]. Both passive leg raising and fluids improved microcirculatory parameters, including perfused capillary density, proportion of perfused vessels, microcirculatory flow index, and heterogeneity index [6]. In the paper published in June, Ospina-Tascon et al. [7] used SDF imaging to evaluate the microcirculation in septic patients before and after fluid resuscitation in different patients early $(<24 \mathrm{~h})$ or late $(>48 \mathrm{~h})$ in their clinical course. Fluid resuscitation increased perfused vessel density early, but not late.

Fluid resuscitation in sepsis starts with identifying patients who are preload-responsive, that is, who are likely to respond to fluids with increased stroke volume [8]. The current studies [4, 7] suggest that fluid resuscitation improves microcirculatory perfusion in ways that are not entirely explained by systemic hemodynamic effects. In the Ospina-Tascon study [7] about half of patients were hemodynamic responders [defined as an increase in mean arterial pressure (MAP) by $>5 \%$, or increase in cardiac output (CO) by $>15 \%$ ], but microcirculatory benefits did not correlate with either MAP or CO. In addition, in patients treated late, there was some improvement in MAP and $\mathrm{CO}$, but no change in the microcirculation. In the Pottecher study [4], change in microcirculatory flow index did correlate with change in CO and MAP, and change in proportion of perfused vessels correlated with changes in CO but not MAP. Readers should be cautious about overinterpreting either correlations or lack thereof in relatively small studies such as these. The main point is not that there is no relationship whatsoever between macrocirculatory and microcirculatory hemodynamics, but rather that there are some independent determinants of flow in different circulatory beds. In addition, there is clearly room for individual variability. A recent study examining the effect of varying perfusion pressure with norepinephrine on responses of the sublingual microcirculation found no 
effect on the population as a whole, but a fair amount of variation in individual responses, with patients reaching maximal perfusion at different mean arterial pressures [9].

These studies also lend credence to the notion that evaluation of microcirculation perfusion might be worthwhile as a clinical index of the adequacy of fluid resuscitation in individual patients. Direct visualization of the sublingual circulation has shown microcirculatory perturbation in patients with sepsis $[10,11]$, and changes appear to track the clinical course $[12,13]$. The study of Ospina-Tascon et al. suggests that some patients without changes in MAP or $\mathrm{CO}$ in response to fluids will still show improvement in the microcirculation. Nonetheless, visualization of the sublingual circulation is not quite ready for routine clinical application at the bedside. As it evolves, this technology needs to become more userfriendly; there is room for improvement in both ease of acquisition and analysis to facilitate generation of reproducible and timely data to guide patient management [6].

Study of the microcirculation might also provide insight into potential mechanisms of disease, something that is hinted at but not fully addressed in the current studies. Local hemodynamics might matter even if global changes in response to therapy are more subtle. The increase in perfused capillary density seen in patients without dramatic changes in cardiac index in the Ospina-Tascon study lends credence to this notion. These authors also found a correlation between changes in the proportion of perfused microvessels and changes in serum lactate, supporting a similar correlation between perfused capillary density and oxygen extraction ratio in sepsis found by Trzeciak et al. [11] and mechanistic data directly relating stopped flow capillaries to oxygen extraction in an animal model of sepsis [14]. This evidence, albeit indirect, supports the notion that perturbation of microcirculatory hemodynamics impairs perfusion at the local level. The studies also provide evidence for increases in perfused capillary density, something that could happen if local pressures were increased to values that exceed their critical closing pressures [15]. Increased cardiac output, which was observed in most of the patients in the Pottecher study and half of the patients in the Ospina-Tascon study, could also potentially mediate local vasodilation by increasing shear stress. Other potential mechanisms include possible influences on blood rheology and plasma viscosity, as well as modulation of the complex humoral and neurologic adaptive responses of microvascular networks to stress.

One intriguing finding of the investigation of OspinaTascon et al. was the difference between microvascular responses early and late in the course of sepsis despite similar increases in global hemodynamic parameters. It seems clear that measures to support hemodynamics after presentation with sepsis are most effective when applied early [16], and the improvement in microcirculation perfusion has been shown to correlate with improvements in organ function in the early phases of sepsis [13]. Whether patients in whom macrocirculatory goals have been achieved should be resuscitated to microcirculatory endpoints has not been established, and is the subject of ongoing investigation [3]. The later phases of sepsis, when multiple organ failure tends to predominate, however, remain a mystery [17]. It is not at all clear that attempts at hemodynamic optimization late will have the same effect as similar attempts early, and the current data suggest they may not. Defining "early" versus "late" phases in the clinical course of sepsis, however, remains a challenge. Ospina-Tascon et al. used $24 \mathrm{~h}$ or less and $48 \mathrm{~h}$ or more, which is reasonable for the purposes of study design, but leaves a period of uncertainty in between for the clinician trying to apply their findings to clinical practice. Given the variability in clinical courses in individual patients, further research in this area might try to move beyond time-based definition of phases to more state-based definitions, in which recognizable characteristics are used to define when an early phase has given way to a later one.

These studies $[4,7]$ have used sophisticated methodology to elucidate microvascular effects of fluid resuscitation, but we still have a lot to learn about the microcirculation in sepsis. In other contexts, we are learning to "think globally, act locally." With respect to our patients with sepsis, we should still be thinking globally (assembling individual pieces of data into a generalized whole to generate a therapeutic plan), but there is a persuasive case to be made that, at least with respect to hemodynamics, it is local actions on the microcirculation that are most important. Learning how to assess the impact of systemic interventions such as fluid resuscitation is only the first step toward developing interventions that target microcirculatory perfusion.

\section{References}

1. Hollenberg SM, Ahrens TS, Annane D, Astiz ME, Chalfin DB, Dasta JF, Heard SO, Martin C, Napolitano LM, Susla GM, Totaro R, Vincent JL, ZanottiCavazzoni S (2004) Practice parameters for hemodynamic support of sepsis in adult patients: 2004 update. Crit Care Med 32:1928-1948
2. Ince C, Sinaasappel M (1999) Microcirculatory oxygenation and shunting in sepsis and shock. Crit Care Med 27:1369-1377
3. Trzeciak S, Cinel I, Phillip Dellinger R, Shapiro NI, Arnold RC, Parrillo JE, Hollenberg SM (2008) Resuscitating the microcirculation in sepsis: the central role of nitric oxide, emerging concepts for novel therapies, and challenges for clinical trials. Acad Emerg Med 15:399-413 
4. Pottecher J, Teboul J-L, Georger J-F, Laplace C, Benhamou D, Vicaut E, Duranteau J (2010) Both passive leg raising and intravascular volume expansion improve sublingual microcirculatory perfusion in severe sepsis and septic shock patients. Intensive Care Med. doi: 10.1007/s00134-010-1966-6

5. Michard F, Boussat S, Chemla D, Anguel N, Mercat A, Lecarpentier Y, Richard C, Pinsky MR, Teboul JL (2000) Relation between respiratory changes in arterial pulse pressure and fluid responsiveness in septic patients with acute circulatory failure. Am J Respir Crit Care Med 162:134-138

6. De Backer D, Hollenberg S, Boerma C, Goedhart P, Buchele G, Ospina-Tascon G, Dobbe I, Ince C (2007) How to evaluate the microcirculation: report of a round table conference.

Crit Care 11:R101

7. Ospina-Tascon G, Nevese AP, Occhipinti G, Donadello K, Buchele G, Simion D, Chierego ML, Silva TO, Fonseca A, Vincent JL, De Backer D (2010) Effects of fluids on microvascular perfusion in patients with severe sepsis. Intensive Care Med 36:949-955. doi: 10.1007/s00134-010-1843-3
8. Antonelli M, Levy M, Andrews PJ, Chastre J, Hudson LD, Manthous C, Meduri GU, Moreno RP, Putensen C, Stewart T, Torres A (2007) Hemodynamic monitoring in shock and implications for management. International consensus conference, Paris, France, 27-28 April 2006. Intensive Care Med 33:575-590

9. Dubin A, Pozo MO, Casabella CA, Palizas F Jr, Murias G, Moseinco MC, Kanoore Edul VS, Palizas F, Estenssoro E, Ince C (2009) Increasing arterial blood pressure with norepinephrine does not improve microcirculatory blood flow: a prospective study. Crit Care 13:R92

10. De Backer D, Creteur J, Preiser JC, Dubois MJ, Vincent JL (2002) Microvascular blood flow is altered in patients with sepsis. Am J Respir Crit Care Med 166:98-104

11. Trzeciak S, Dellinger RP, Parrillo JE, Guglielmi M, Bajaj J, Abate NL, Arnold RC, Colilla S, Zanotti S, Hollenberg SM (2007) Early microcirculatory perfusion derangements in patients with severe sepsis and septic shock: relationship to hemodynamics, oxygen transport, and survival. Ann Emerg Med 49:88-98 98 e81-e82
12. Sakr Y, Dubois MJ, De Backer D, Creteur J, Vincent JL (2004) Persistent microcirculatory alterations are associated with organ failure and death in patients with septic shock. Crit Care Med 32:1825-1831

13. Trzeciak S, McCoy JV, Phillip Dellinger R, Arnold RC, Rizzuto M, Abate NL, Shapiro NI, Parrillo JE, Hollenberg SM (2008) Early increases in microcirculatory perfusion during protocol-directed resuscitation are associated with reduced multi-organ failure at $24 \mathrm{~h}$ in patients with sepsis. Intensive Care Med 34:2210-2217

14. Ellis CG, Bateman RM, Sharpe MD, Sibbald WJ, Gill R (2002) Effect of a maldistribution of microvascular blood flow on capillary $\mathrm{O}_{2}$ extraction in sepsis. Am J Physiol Heart Circ Physiol 282:H156-H164

15. Pries AR, Secomb TW (2003) Rheology of the microcirculation. Clin Hemorheol Microcirc 29:143-148

16. Task Force of the American College of Critical Care Medicine, Hollenberg SM, Ahrens TS, Astiz ME, Chalfin DB, Dasta JF, Heard SO, Martin C, Susla GM, Vincent JL (1999) Practice parameters for hemodynamic support of sepsis in adult patients. Crit Care Med 27:639-660

17. Singer M, De Santis V, Vitale D, Jeffcoate W (2004) Multiorgan failure is an adaptive, endocrine-mediated, metabolic response to overwhelming systemic inflammation. Lancet 364:545-548 